\title{
Beaconless Pointing for Deep-Space Optical Communication
}

\author{
Aaron J. Swank* $\quad$ Eliot Aretskin-Hariton ${ }^{\dagger} \quad$ Dzu K. Le ${ }^{\dagger} \quad$ Obed S. Sands ${ }^{\ddagger}$ \\ Adam Wroblewski § \\ NASA Glenn Research Center, Cleveland, OH, 44135
}

\begin{abstract}
Free space optical communication is of interest to NASA as a complement to existing radio frequency communication methods. The potential for an increase in science data return capability over current radio-frequency communications is the primary objective. Deep space optical communication requires laser beam pointing accuracy on the order of a few microradians. The laser beam pointing approach discussed here operates without the aid of a terrestrial uplink beacon. Precision pointing is obtained from an on-board star tracker in combination with inertial rate sensors and an outgoing beam reference vector. The beaconless optical pointing system presented in this work is the current approach for the Integrated Radio and Optical Communication (iROC) project.
\end{abstract}

\section{Introduction}

$\mathrm{P}$ ROVIDING communication services to space missions throughout the solar system is the responsibility of the NASA Space Communication and Navigation (SCaN) Program. ${ }^{1}$ A primary goal for SCaN is to develop a communications infrastructure for NASA with the highest data rates feasible in order to ensure the scientific capability of space missions is not limited by the communications infrastructure. Optical communications systems, as compared to traditional radio frequency (RF) communication methods, promise a substantial increase in data return with a corresponding savings in mass, power and volume requirements. ${ }^{2}$ Therefore, free-space optical communication is of interest to NASA to complement radio frequency communication for the potential increase in science data return capability. As a result, the SCaN network architecture features "aggressive, yet systematic, infusion of optical communications to complement the RF baseline". ${ }^{1}$

Despite the potential advantages presented by optical systems, free-space optical communication is not absent of technological challenges. For example, RF communication is transmitted in a broad beam illuminating a target area, whereas optical communication utilizes a comparatively narrow beam, pointed at a single receiver. While the narrow optical beam results in more efficient use of the electromagnetic spectrum as compared to RF, free-space optical communication is challenged by more stringent pointing and tracking requirements due to optical beam divergence. State-of-practice methods for space-based optical communication rely on closed-loop tracking of an Earth-based optical beacon in order to maintain the satellite optical terminal pointing direction towards the ground-station receiver. The Lunar Laser Communication Demonstration (LLCD), for example, has demonstrated that a beacon-aided laser communications technique is feasible for near to Earth orbiting satellites. ${ }^{3,4}$ Yet for deep space optical communication, such as from Mars or beyond, beacon-aided optical communication becomes less practical as the distance increases. ${ }^{2}$ As a result, alternatives to beacon-based laser communications are being investigated. ${ }^{2,5-7}$

The work presented here is motivated in support of the optical communications system for the Integrated Radio and Optical Communication (iROC) project. The iROC project is a research and development effort to couple deep space radio frequency and optical communication elements into an integrated system for communication to Earth at distances from Mars and beyond. iROC enables insertion of optical communication

\footnotetext{
*Research Engineer, Information and Signal Processing Branch

$\dagger$ Aerospace Technologist Control Engineer, Intelligent Control and Autonomy Branch

¥Research Electronics Engineer, Information and Signal Processing Branch

$\S$ Research Engineer, Optics and Photonics Branch
} 


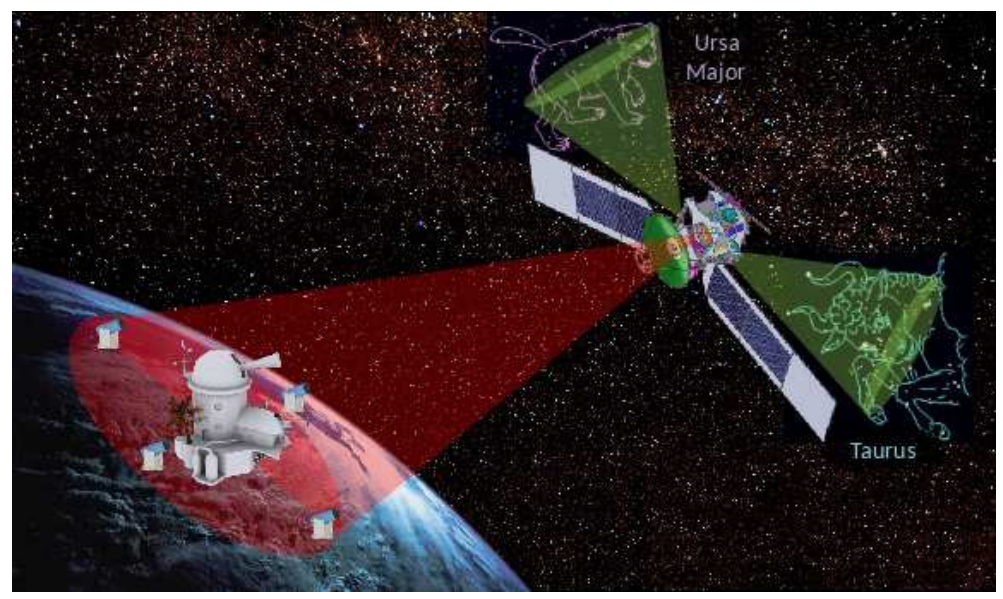

Figure 1. Beaconless pointing for deep-space optical communication.

technology into the NASA communication infrastructure with reduced infusion risk, while leveraging the distinct advantages that both RF and optical techniques provide. The iROC system utilizes a combined RF and optical teletenna (telescope/antenna) to produce a co-boresighted microwave and optical communications system. Additional information on the iROC project and overall design is available in Raible et. al. ${ }^{2}$

\section{A. Beaconless Pointing}

For precision pointing of the optical communications laser, the iROC project is investigating the use of a beaconless pointing approach, whereby no uplink beacon is used (Figure 1). While a beacon-aided technique is likely sufficient for a number of applications, an uplink beacon technique is problematic for deep space applications. High intensity laser power is required to reach the extents of deep space and the satellite telescope design requires a large aperture to receive a dim uplink beacon with sufficient intensity for detection. The technique is further complicated by terrestrial cloud obscuration. ${ }^{8}$ Furthermore, the high intensity uplink beacon poses usage constraints with respect to airspace penetration to avoid air traffic disruption (e.g. for eye safety issues), as well as risk mitigation to orbiting satellites with Earth observing payloads. ${ }^{8,9}$ Although the advantages of a beaconless pointing approach to optical communication have been identified, a beaconless optical communication system has yet to be demonstrated. As a result, the Interagency Operations Advisory Group (IOAG), Optical Link Study Group (OLSG), which was setup to make an intensive study on the operation of optical communications, concluded as a level two priority that "in parallel to the beacon standardization, the space agencies should conduct a rigorous detailed study ... to determine if there are ways of accomplishing beaconless pointing, acquisition and tracking (PAT)". ${ }^{9}$ In addition to motivations outlined by the OLSG, the potential advantages of the beaconless pointing approach include:

- beacon receive requirements imposed on spacecraft are eliminated

- permits optimization of aperture, laser power and pointing knowledge ${ }^{2}$ (e.g. no minimum telescope aperture size required to detect a dim beacon)

- operation is independent of beacon cloud obscuration resulting in increased spacecraft autonomy

- high power uplink beacon not required

- eliminates eye safety concerns from uplink beacon; laser clearing house coordination not necessary

It is therefore desirable to consider alternate designs, which do not utilize the uplink beacon to aid the optical pointing system. The following sections outline the current baseline approach to the iROC beaconless pointing system. 


\section{Methodology}

Operating a deep-space optical communications system in a beaconless fashion is challenging because the system is operating open loop without feedback of the pointing error directly from the receiving ground station. An uplink beacon, for example, would close the feedback loop and provides the necessary relative offset information to correct the pointing direction vector. In absence of an uplink beacon, pointing error feedback information must be generated using sensors on-board the spacecraft. As a result, the beaconless approach requires a precise and absolute pointing direction estimate for the outgoing beam. Furthermore, the desired pointing direction for the outgoing beam must be maintained by the pointing control system. In general, the pointing subsystem components required to establish a beaconless optical communication link may be summarized to include: A) optical platform attitude estimation, B) outgoing beam direction vector estimation, C) optical line of sight stabilization, and D) system control.

\section{A. Optical Platform Attitude Estimation}

Optical platform attitude estimation requires either relative attitude information from a known pointing direction, or absolute pointing knowledge coupled with target location information. Sources for optical beam pointing direction information, which do not utilize an artificial uplink beacon, may be grouped into two general categories: 1. using Earth signatures as a virtual beacon (at various wavelengths) for relative pointing information, and 2. using celestial sources (e.g. stars) and inertial sensors for absolute attitude information. Concepts using signatures of the Earth as a virtual beacon source has been discussed in the literature for both the visible and long-wave infra-red regimes. ${ }^{10-13}$ Regardless of the observed wavelength, using the Earth for line of sight vector information has an issue with non-uniformity of the signal and limited signal strength. As a result, signal centroiding will not necessarily coincide with the geometric center of the Earth, limiting the accuracy of the approach. As an alternative, celestial sources can provide attitude and pointing information by using star tracker devices. Star trackers provide very accurate absolute pointing information, with precision on the order of a microradian and advanced technologies reaching the sub microradian range. Further advancements in star tracker technology is expected with improved technologies, including sensor technology and maturation of interferometric star tracker methods ${ }^{14,15}$ as well as significantly improved star catalog information (over $100 \times$ increase in accuracy) using precision astrometry results from the European Space Agency (ESA) Gaia mission to reach accuracies better than 100 picoradians. ${ }^{16-18}$

For establishing beaconless optical communication from Mars to Earth, the iROC design ${ }^{2}$ requires an optical beam pointing accuracy on the order of 2-5 $\mu \mathrm{rad}$. Precision attitude information, combined with satellite state information, is sufficient for optical beam pointing information. Using celestial sources to generate the optical platform attitude estimate is the baseline approach of the iROC project. Here, a star tracker and an Inertial Reference Unit (IRU) are co-located on the optical bench. The star tracker information is combined with the high-rate IRU information for direct attitude determination of the optical platform. In contrast to other systems requiring precision attitude information for scientific payloads, attitude information originates from the optical bench platform rather than from sensors located on the spacecraft. As a result, the configuration does not require methods to measure and correct for the relative motion between the spacecraft attitude sensors and optical bench, such as an elaborate metrology system or reverse kinematic estimation algorithms for the pointing gimbal mechanism. In this application the IRU gyroscope information provides relative change in attitude information through either a delta angle or angular rate value. The gyroscope angular rate (or delta angle) information is used to propagate the optical platform attitude at a rate sufficiently high to capture the optical platform dynamics. Currently it is estimated that an output rate of several hundred $\mathrm{Hz}$ is sufficient for the iROC application. A multiplicative Extended Kalman Filter (EKF) corrects for the gyro drift and estimates an absolute pointing direction of the optical platform using the star tracker information and the propagated attitude estimate from the gyroscopes ${ }^{\mathrm{a}}$. The EKF is updated at the rate of the star tracker measurement rate. Details of the EKF formulation is out of the scope of this paper, yet detailed information on the multiplicative EKF approach for attitude estimation is widely published. ${ }^{19-23}$

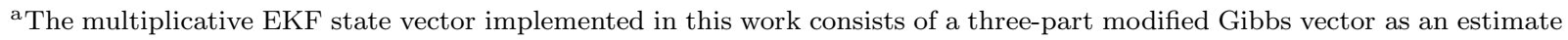
of attitude and a three-part gyro bias estimate. 


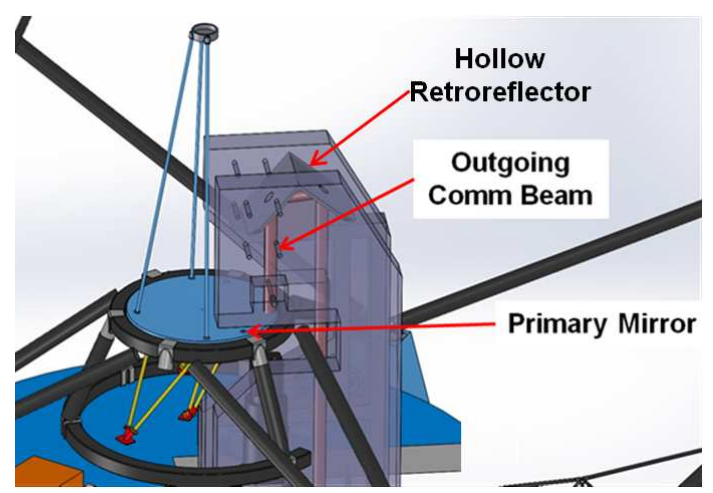

(a) Notional iROC optical assembly

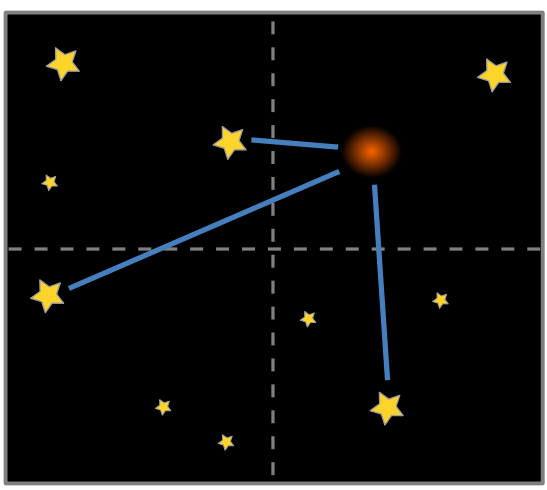

(b) Outgoing beam shown with star field.

Figure 2. Fig. 2(a): Star tracker sensor mounted in a notional iROC assembly for direct measurement of the outgoing beam direction. A fraction of the outgoing communications beam is redirected into the interferometric star tracker. Fig. 2(b): Effective view field of interferometric star tracker. The angular differences (blue) between outgoing communications beam (red circle) and reference stars are on a common sensor.

\section{B. Outgoing Beam Direction Vector Estimation}

Determining the direction of the outgoing communication laser beam is a fundamental challenge of the beaconless pointing approach. Since the desired line of sight vector is determined by knowledge of the receive station location, the beam direction vector must be related to an inertial pointing direction. Determining the optical beam direction vector in inertial space typically requires a two-part approach, where one first measures the attitude of the optical platform in inertial space (such as through utilization of a star tracker device), followed by estimating the beam's direction vector relative to the optical platform. Although the optical transmitter is designed with a high stiffness and low coefficient of thermal expansion material, the optical assembly is subject to spatial deformation due to structural and thermal loads induced by a variety of sources. For an accurate estimate of the beam direction vector, the design requires a method to measure the relative motion between the attitude reference sensor and the communication beam. Disturbances such as vibrational motion and thermal stability of structure and optical mounting components will contribute to beam motion, which is not common to the attitude information provided by the attitude sensor. For the design presented here, the problem is simplified (though not eliminated) by locating the star tracker on the optical bench.

A proposed approach for estimating the beam direction vector involves a direct measurement of the outgoing beam direction with the star tracker sensor. Here, the star tracker detector is designed to be sensitive in the short wave infra-red (SWIR) band to allow direct sensing of the $1550 \mathrm{~nm}$ communication laser and the star light on a common detector. As shown in Figure 2, the concept samples a small portion of the outgoing communication laser beam through the use of a hollow retro-reflector. Through exploitation of a hollow retro-reflector to sample the laser light after reflection from the primary mirror, the angular direction of the outgoing beam can be estimated since it is located along a vector parallel to the communication beam. This sampled beam is directed into the optics of the star tracker system for angular measurement of the beam direction. By directly measuring the outgoing optical beam direction after any imposed disturbances, the measurement directly relates the star tracker attitude information to the laser beam line of sight direction vector through a common sensor. Angular measurement of the incoming beam is possible through the use of an interferometric star tracker device. ${ }^{14,15}$ The interferometric measurement nature of the star tracker device is a key characteristic of the star tracker hardware necessary to implement this design. An interferometric star tracker measures the incoming angle of the star/laser source rather than inferring an incoming angle through a centroiding technique typical of other star trackers. With an interferometric star tracker, a change in the light incident angle results in changes to a phase pattern, which allows for precise detection of angular motion. Thus, by using an interferometric star tracker, the relative angular position between the laser beam source and the star light source is precisely determined by a phase estimate calculation resulting in improved accuracy as compared to a centroiding technique. In the current proposed design currently under development by the Optical Physics Company, the interferometric star tracker performance is expected to 


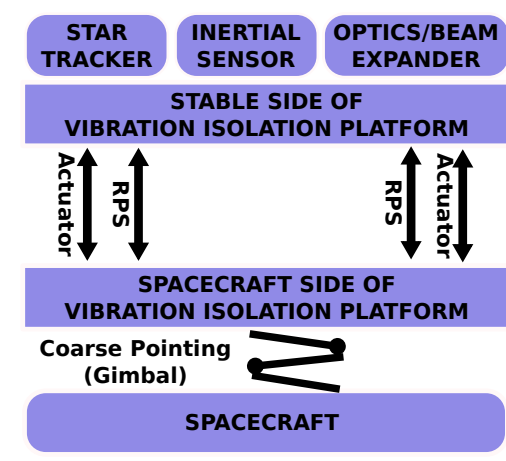

(a) Block Diagram

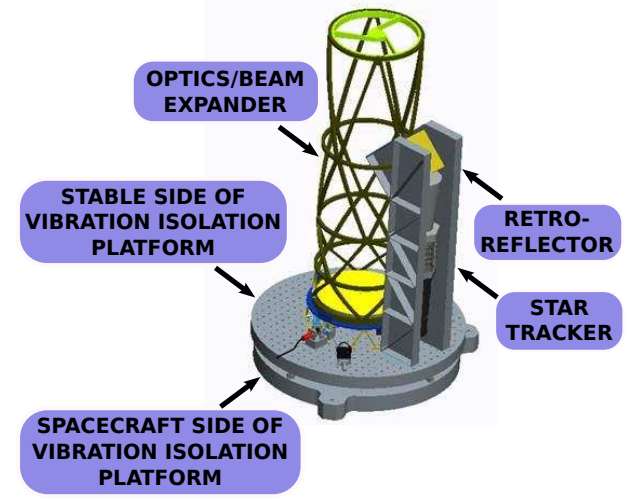

(b) Solid Model

Figure 3. Stabilized platform beaconless pointing system. Fig. 3(a): The vibration isolation platform provides disturbance suppression and fine steering capability for the optical beam. Fig. 3(b): Solid model rendering of conceptual stabilized platform system. The coarse pointing (i.e. gimbal) assembly is not depicted in the solid model. Also not visible are the vibration isolation platform sensors and actuators, which are located between the stable and spacecraft side of the vibration isolation platform.

achieve better than $0.3 \mu \mathrm{rad}$ attitude determination at $15 \mathrm{~Hz}$.

\section{Optical Line of Sight Stabilization}

The ability to stabilize and point the outgoing communications beam is an essential feature of a space optical communication terminal. Disturbances to the pointing direction will be a result of a number of contributions. For example, in addition to spacecraft induced structural vibrational motion, thermal stability of structure and optical mounting components will also contribute to beam motion. Mechanical disturbances from the host spacecraft and thermal beam wander must be accommodated with beam stabilization mechanisms integrated into the iROC terminal. Requirements for beam pointing accuracy and beam stabilization follow from requirements on power flux density of the transmit beam at the receive terminal and set the overall pointing accuracy of the outgoing communications beam somewhere between 2-5 $\mu$ rad noise equivalent angle (NEA). For the iROC system, generic requirements on the input mechanical disturbance profile at the spacecraft interface are assumed as per past and current deep-space optical communications development efforts. It is assumed here that the spacecraft design will not be optimized to reduce the disturbance imparted on the optical communications system. For design purposes, a nominal (and conservative) spacecraft disturbance environment is assumed. Angular power spectral density with total integrated disturbance levels in the range of $100-150 \mu \mathrm{rad}$ root mean squared (RMS) are adopted for iROC. ${ }^{24,25}$ Both three-axis rotational mechanical disturbances and three-axis translational mechanical disturbance are included in the requirements set. The iROC design should, therefore, be applicable to a wide range of spacecraft hosts.

In order to achieve the desired pointing performance of $2-5 \mu \mathrm{rad}$ for the iROC system, measures must be taken to stabilize the optical line of sight. For the iROC system, a beaconless stabilized platform approach is assumed for the communications terminal. As noted by Chen et. $\mathrm{al}^{26}$ the stabilized platform approach eliminates the need for high bandwidth beam stabilization control loop, including fast steering mirrors and detectors. Recent innovations in component technologies such as star trackers and vibration isolation enable the approach. The key components of the stabilized platform approach are schematically depicted in Figure 3. The design consists of the iROC terminal, including the optical assembly and beam expander, located on a vibration isolation platform. The spacecraft side of the vibration isolation platform is connected to the spacecraft interface through a coarse-pointing mechanism such as a gimbal assembly. Located on the stable side of the vibration isolation platform, is an absolute attitude sensor (e.g. the star tracker) and an inertial sensor (e.g. an IRU or gyroscope) for high-rate angle or angular rate information. The vibration isolation platform not only reduces satellite vibrational disturbance contributions to the outgoing optical beam, but also stabilizes the star tracker co-located on the optical bench. As a result, reduced jitter is imparted on the star tracker focal plane array for improved attitude sensing. The stable side of the vibration 


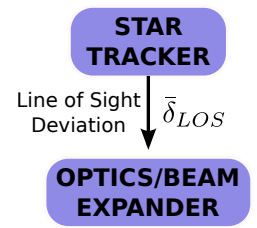

(a)

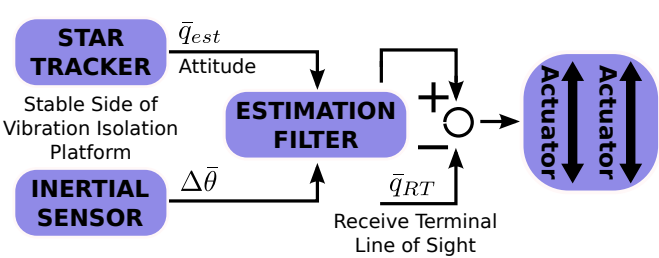

(b)

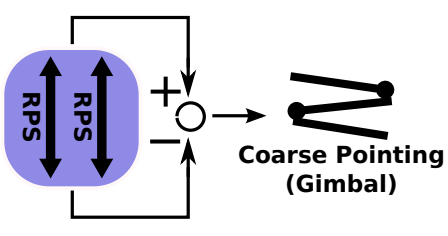

(c)

Figure 4. Stabilized platform control architecture. Fig. 4(a): Star tracker and outgoing communications beam alignment loop. The star tracker also provides updates to the outgoing beam direction vector estimates, or line of sight deviations. Fig. 4(b): Platform jitter rejection and fine-pointing control loop. The estimation filter, e.g. EKF, provides an attitude for the stable side of the vibration isolation platform, which is used for platform actuation. Fig. 4(c): Coarse pointing control loop. Coarse pointing of the platform is achieved by a gimbal and driven by relative angular position sensor information.

isolation platform is connected to the spacecraft side of the vibration isolation platform using a hinge joint or other motion-constraining device such as a flexure. Electro-mechanical actuators and relative angular position sensor (RPS) are located between the stable and spacecraft side of the vibration isolation platform for feedback control. The vibration isolation platform also supplies the fine pointing capability to the system, with microradian pointing control and stroke on the order of a milliradian. In the current design, emphasis is placed on disturbance suppression for the iROC terminal with minimal active beam control by steering devices. In the event that platform stabilization is not sufficient through laboratory testing, the design can be augmented with a high-bandwidth laser metrology system coupled with fast steering mirrors for mechanical jitter suppression. The resulting system would then be a hybrid, consisting of both beam stabilization and platform stabilization.

\section{System Control}

A simplified representation of the control loops for the stabilized beaconless platform is depicted in Figure 4. As described in Section B, registration of the outgoing communications beam to the star tracker attitude information is accomplished by feeding a portion of the outgoing communications beam into the star tracker. Deviations in the location of the outgoing communications beam serve as an error signal to actuator(s) within the optical assembly to provide precise, low-rate control (at the star tracker update rate and below) of the outgoing beam line of sight. The beam-alignment loop, as shown in Figure 4(a), compensates for effects such as thermal beam wander and other slowly varying effects which contribute to beam line of sight deviation. Pointing system calibration is performed periodically by dithering the beam with small pointing offsets around the receiver location and measuring changes in received power with offsets in commanded beam pointing offsets. The received power levels are fit to commanded pointing offsets in order to determine the center of the beam pattern. The nominal communications beam position in the star tracker is then updated to accommodate for structural changes.

Platform pointing and jitter rejection is accomplished through the control loop as shown in Figure 4(b). Platform attitude information from the star tracker is combined with inertial sensor (IRU or gyro) information using the EKF approach as described in Section A to yield high bandwidth stable-side attitude at the gyro update rate. The resulting attitude estimate is compared (subtracted) with the receive terminal line of sight vector and used to drive the isolation platform actuators for pointing and jitter rejection. Calculation of the receive terminal line of sight vector must accommodate receive terminal motion occurring during the time of flight. As shown in Figure 4(c), control of the coarse pointing gimbal is derived from the RPS information, which measures the relative angle between the spacecraft and stable side of the vibration isolation platform. The coarse pointing gimbal offloads and decouples stable side pointing of the vibration isolation platform. By maintaining separate, decoupled control loops for a) beam alignment, b) jitter-rejection and fine pointing, and c) coarse pointing, the stabilized platform approach simplifies the design and analysis. 
Table 1. IRU noise terms used for simulations.

\begin{tabular}{cccc}
\hline & \multicolumn{2}{c}{ Gyro Class: } \\
Nomenclature & Units & Navigation & Strategic \\
\hline \hline Angle Random Walk & {$\left[\mathrm{deg} / \mathrm{h}^{1 / 2}\right]$} & $8 \mathrm{e}-4$ & $0.5 \mathrm{e}-4$ \\
Bias Instability & {$[\mathrm{deg} / \mathrm{h}]$} & $1 \mathrm{e}-2$ & $1 \mathrm{e}-3$ \\
\hline \hline
\end{tabular}

Table 2. Star tracker noise equivalent angle values assumed for simulations.

\begin{tabular}{cccc}
\hline Nomenclature & Units & ST5000 $^{27}$ & Advanced ST \\
\hline \hline Cross-Boresight NEA & {$[\mu \mathrm{rad}]$} & 2.6 & 0.4 \\
Boresight NEA & {$[\mu \mathrm{rad}]$} & 82.4 & 2.0 \\
Sample Rate & {$[\mathrm{Hz}]$} & 10 & 15 \\
\hline \hline
\end{tabular}

\section{Simulation}

To assess the capabilities of the attitude solution created by a combined star tracker and IRU, an Extended Kalman Filter software simulation was implemented. The performance of the EKF tool is first verified using simulation parameters representative of a test scenario for which results are available. After verification of the tool, the EKF performance is simulated for different star tracker and IRU configurations. The assumed values for the IRU and star tracker used for the simulations is compiled into Table 1 and Table 2. For simulated star tracker measurements, Gaussian white noise is added to a truth trajectory consisting of a sinusoidal varying rotation at $100 \mu \mathrm{rad} / \mathrm{s}$. To simulate an IRU measurement for the attitude determination simulations, a time series angular rate truth value is generated with simulated noise consisting of gyro angular random walk (ARW) and bias instability. Since the anticipated dynamics are small, the gyro scale factor stability is not considered as the errors are dominated by gyro drift. The angle error growth is dominated over short time periods by ARW and over long time periods by the bias instability. Realistic gyro noise values are generated by shaping the spectral content of a Gaussian white noise sequence in the frequency domain using power spectral density constructed from the asymptotic components as described in the IEEE Standard $952 .{ }^{28}$

Verification of the EKF simulation is performed using hardware specifications similar to the Wallops Arc Second Pointer (WASP) system, as flight performance data is published online. ${ }^{29}$ Initial WASP test flights used an LN-251 IRU produced by Northrop Grumman and the ST5000 star tracker produced by the University of Washington. ${ }^{27}$ Key performance metrics of the LN-251 for the bias instability and ARW are not published. As a result, values representative of a navigation grade gyro are assumed in the simulation. The performance characteristics for the NEA of the ST5000 is published ${ }^{27}$ and used in the simulations.

Performance results of the navigation class gyro and ST5000 combination have been produced by several WASP test flights, the most applicable to the baseline EKF scenario presented here is test flight two. Test flight two was performed with the star tracker and IRU in the control loop. While the EKF designed for the simulation detailed in this paper contains only an attitude solution, it is assumed that adding a control loop can only degrade, not improve, the final attitude solution. Results from the second WASP test flight show an attitude error on the order of 0.5 and 0.75 arcseconds (3-sigma) in the pitch and yaw respectively. This corresponds to an attitude error of $0.8 \mu \mathrm{rad}$ RMS in the pitch and $1.2 \mu \mathrm{rad}$ RMS in the yaw. The results closely match the predicted value from the EKF simulation shown in Figure 5 which shows an attitude error of $1.15 \mu \mathrm{rad}$ RMS in yaw and pitch. These results validate the EKF simulation as the results are on the same order as the expected flight capabilities of a given star tracker and IRU combination.

\section{Discussion and Results}

As an example of the attitude determination performance expected to be available for iROC, a simulation of the EKF is performed with star tracker and inertial reference unit parameters representative of high 

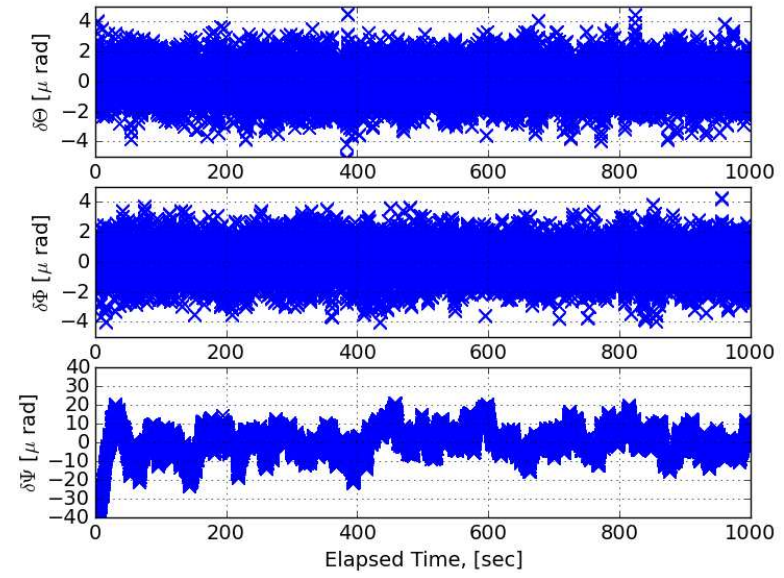

(a) Attitude error from truth.
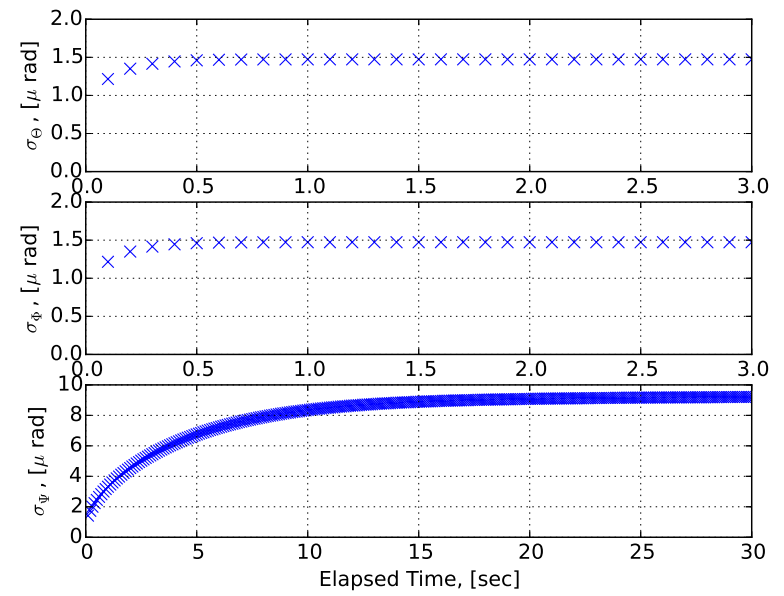

(b) EKF modified Gibbs vector covariance estimate.

Figure 5. Attitude error from truth and Kalman filter Gibbs vector covariance estimate. The RMS error from truth is $1.15 \mu \mathrm{rad}$ in the cross boresight directions $(\Theta, \Phi)$ and $8.35 \mu \mathrm{rad}$ around the boresight direction ( $\Psi)$. The extended Kalman filter converges in the cross boresight directions $(\Theta$, $\Phi)$ within approximately 0.5 sec and within 20.0 sec for the boresight direction $(\Psi)$.

performance devices. With the advent of interferometric star tracker technologies, one expects advanced star tracker performance to reach the sub-microradian accuracies. For the inertial reference unit, a device with performance at the strategic grade gyro level is used. A strategic grade gyro and a next generation advanced star tracker will provide an upper bound to the expected attitude estimation performance. The hardware noise and performance values are provided in Table 1 and Table 2. The ARW value listed in the table for the strategic grade gyro follows published values for the Scalable Inertial Reference Unit (SIRU) for space manufactured by Northrop Grumman. ${ }^{30}$ Since the bias instability is not directly published for the SIRU, a representative value is used. It is worth noting that current commercially available strategic grade gyros tend to be larger and require more power than IRU's of lower performance. The SIRU for example is listed at $43 \mathrm{~W}$ max power and a mass of $7.1 \mathrm{~kg} .{ }^{30}$ While such mass and power requirements are problematic for the iROC application, advances in IRU technology such as the milli-Hemispherical Resonator Gyro (HRG) by Northrop Grumman ${ }^{31}$ present potential improvement in the mass and power requirements without a significant compromise in performance. While the milli-HRG is not yet commercially available, laboratory tests have demonstrated performance values of $3.5 \mathrm{e}-4 \mathrm{deg} / \mathrm{h}$ bias instability and an ARW of $3 \mathrm{e}-4 \mathrm{deg} / \mathrm{h}^{1 / 2}$ with an estimated IRU mass, power and volume of $0.752 \mathrm{~kg}, 5 \mathrm{~W}$, and $377 \mathrm{~cm}^{3}$ respectively. ${ }^{31}$

In the first simulation, a strategic grade IRU is paired with an advanced star tracker with an accuracy of $0.4 \mu \mathrm{rad}$ in the cross boresight direction and $2 \mu \mathrm{rad}$ around the boresight direction. The results of the EKF simulation are depicted in Figure 6. From the time series data of attitude error, as computed in reference to the simulated truth trajectory, the RMS error is $0.14 \mu \mathrm{rad}$ in the cross boresight directions and $0.33 \mu \mathrm{rad}$ around the boresight direction. By inspection of the EKF covariance matrix components, it is seen that the attitude estimate for the cross boresight direction converges in approximately $0.5 \mathrm{sec}$. Due to the simulated reduced precision for the star tracker around the boresight direction, the attitude estimate for the direction along the star tracker boresight takes slightly longer to converge, yet is within approximately 2.0 sec.

For comparison purposes of expected attitude estimation accuracy, a second simulation is also performed with the advanced star tracker and the navigation grade gyro. The results of the EKF simulation are depicted in Figure 7. From the time series data of attitude error, as computed in reference to the simulated truth trajectory, the RMS error is $0.36 \mu \mathrm{rad}$ in the cross boresight directions and $0.99 \mu \mathrm{rad}$ around the boresight direction. By inspection of the EKF covariance matrix components, it is seen that the attitude estimate converges within a few iterations of the filter. In comparison to the simulation performed with the same advanced star tracker, the change from a strategic grade gyro to the navigation grade gyro results in approximately a factor of 3 performance decrease along the boresight direction and a 2.5 factor decrease in the cross boresight direction. Thus, by using the advanced star tracker the iROC project can potentially use a smaller, lighter, and less expensive IRU and still achieve the overall pointing accuracy of 2-5 $\mu \mathrm{rad}$. 


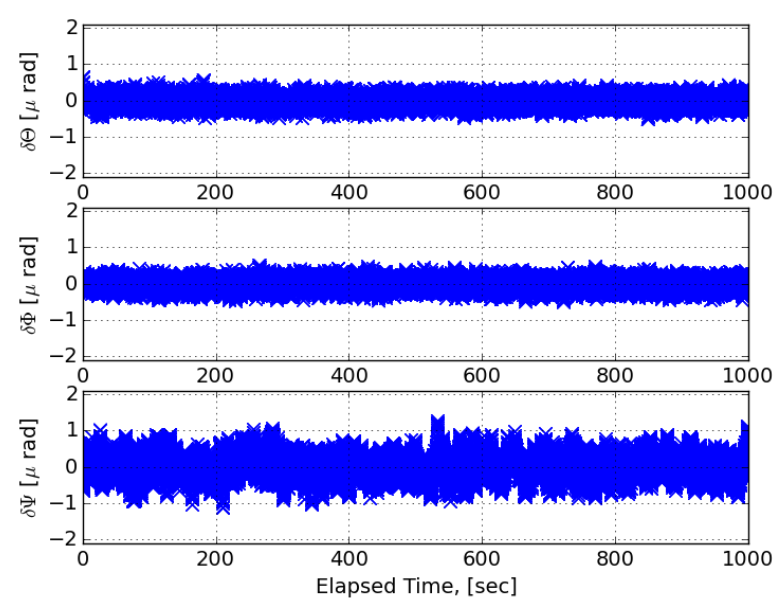

(a) Attitude error from truth.

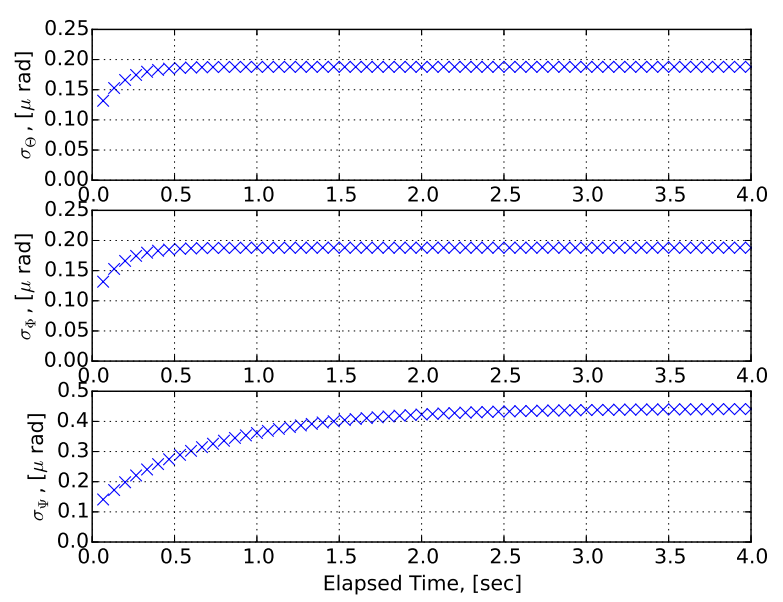

(b) EKF modified Gibbs vector covariance estimate.

Figure 6. Attitude error from truth and Kalman filter Gibbs vector covariance estimate. Simulation uses a strategic grade gyro paired with an advanced star tracker. The RMS error from truth is $0.14 \mu \mathrm{rad}$ in the cross boresight directions $(\Theta, \Phi)$ and $0.33 \mu \mathrm{rad}$ around the boresight direction $(\Psi)$. The extended Kalman filter converges in the cross boresight directions $(\Theta, \Phi)$ within approximately 0.5 sec and within 2.0 sec for the boresight direction $(\Psi)$.
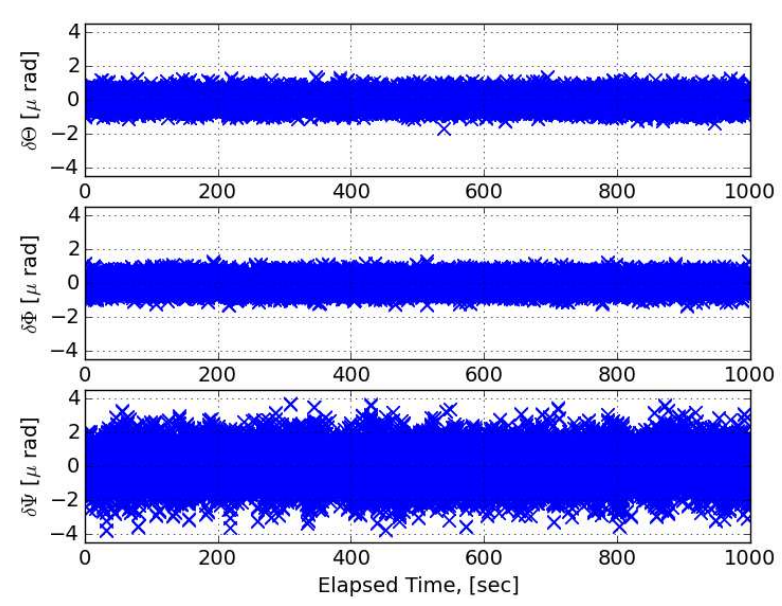

(a) Attitude error from truth.

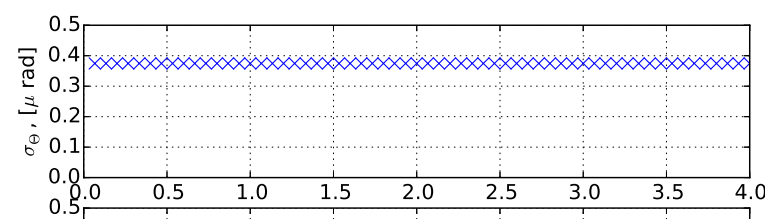

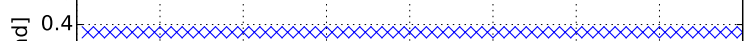

0.3

$\stackrel{3}{*} 0.2$

0.1
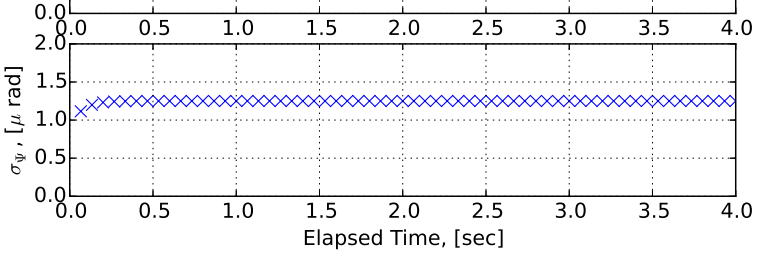

(b) EKF modified Gibbs vector covariance estimate.

Figure 7. Attitude error from truth and Kalman filter Gibbs vector covariance estimate. Simulation uses a navigation grade gyro paired with an advanced star tracker. The RMS error from truth is $0.36 \mu \mathrm{rad}$ in the cross boresight directions $(\Theta, \Phi)$ and $0.99 \mu \mathrm{rad}$ around the boresight direction $(\Psi)$. The extended Kalman filter converges in the cross boresight and boresight directions within a couple of iterations. 


\section{Conclusion}

A beaconless pointing approach for a combined optical and radio frequency system was described. As noted by the Optical Link Study Group, the component technology has developed to the point of beaconless consideration. ${ }^{9}$ The proposed system leverages near-term advances in star tracker technology and a navigation grade IRU. An Extended Kalman Filter pointing simulation was conducted which yields a pointing accuracy of $0.4 \mu \mathrm{rad}$ RMS for each cross boresight axis. This pointing accuracy is sufficient to support an overall pointing system accuracy of $2-5 \mu \mathrm{rad}$ which is required for a deep space beaconless communication. The interferometric star tracker approach uses a single instrument to detect the outgoing communication beam direction relative to the absolute attitude solution. The beam steering control loop can then be closed without a beacon, eliminating beacon receive requirements imposed on the spacecraft while expanding communication availability. Beaconless pointing for deep space optical communication is being further investigated for the iROC project to enable high data rate downlink from Mars and beyond.

\section{Acknowledgements}

This work was supported by the Advanced Communications and Navigation Division within the NASA Space Communications and Navigation (SCaN) Program.

\section{References}

\footnotetext{
${ }^{1}$ Younes, B., Perko, K., and Schier, J., "Space Communications and Navigation (SCaN) Network Architecture Definition Document (ADD) Volume 1: Executive Summary," October 2011.

${ }^{2}$ Raible, D., Romanofsky, R., Budinger, J., Nappier, J., Hylton, A., Swank, A. J., and Nerone, A. L., "On the Physical Realizability of Hybrid RF and Optical Communications Platforms for Deep Space Applications," 32nd Inernational Communications Satellite Systems Conference, AIAA, San Diego, CA, 2014.

${ }^{3}$ Boroson, D. M., Robinson, B. S., Murphy, D. V., Burianek, D. A., Khatri, F., Kovalik, J. M., Sodnik, Z., and Cornwell, D. M., "Overview and results of the Lunar Laser Communication Demonstration," Vol. 8971, International Society for Optics and Photonics, 2014.

${ }^{4}$ NASA, "NASA Laser Communication System Sets Record with Data Transmission to and from Moon," http://www. nasa.gov/press/2013/october/nasa-laser-communication-system-sets-record-with-data-transmissions-to-and-from, Oct 2013, [Online; accessed 22-Oct-2015].

${ }^{5}$ Ortiz, G. G. and Lee, S., "Star tracker based ATP system conceptual design and pointing accuracy estimation," Lasers and Applications in Science and Engineering, International Society for Optics and Photonics, 2006.

${ }^{6}$ Lee, S., Alexander, J. W., and Ortiz, G. G., "Submicroradian pointing system design for deep-space optical communications," 2001.

${ }^{7}$ Lee, S., Ortiz, G. G., and Alexander, J. W., "Star tracker-based acquisition, tracking, and pointing technology for deep-space optical communications," Interplanetary Network Progress Report, Vol. 42, No. 161, 2005, pp. 42-161.

${ }^{8}$ Optical Link Study Group (OLSG), "Optical Link Study Group Final Report," Tech. Rep. IOAG.T.OLSG.2012.V1, Interagency Operations Advisory Group, June 2012.

${ }^{9}$ Optical Link Study Group (OLSG), "Optical Link Study Group Addendum to Final Report," Tech. Rep. IOAG.T.OLSG.2012.V1A, Interagency Operations Advisory Group, November 2012.

${ }^{10}$ Quadrelli, M. B. and Piazzolla, S., "Spacecraft Line-of-sight Stabilization using LWIR Earth Signature," $A I A A S P A C E$ 2012 Conference \& Exposition, 2012.

${ }^{11}$ Biswas, A., Piazzolla, S., Peterson, G., Ortiz, G., and Hemmati, H., "The Long-Wave Infrared Earth Image as a Pointing Reference for Deep-Space Optical Communications," Interplanetary Network Progress Report, Vol. 42, No. $167,2006$.

${ }^{12}$ Lee, S., Ortiz, G., Roberts, W., and Alexander, J., "Feasibility study on acquisition, tracking, and pointing using Earth thermal images for deep-space Ka-band and optical communications," Interplanetary Network Progress Report, Vol. 42, No. $155,2003$.

${ }^{13}$ Marino, B. E., Tsou, H., and Yan, T.-Y., "Use of cross-validation and Monte Carlo simulation for estimating the parameters of beaconless free-space optical pointing and tracking," 2002.

${ }^{14}$ Hutchin, R. A., "Interferometric tracking device," US Patent 8045178, Optical Physics Company, October 2011.

${ }^{15}$ Hutchin, R. A., "Two axis interferometer tracking device and method," US Patent 9297880, Optical Physics Company, March 2016.

${ }^{16}$ Sarri, G., Prusti, T., and Schnorhk, A., "ESA's Billion-Star Surveyor: Gaia Ready for Launch Campaign," ESA Bulletin, Vol. 155, August 2013, pp. 2-11.

${ }^{17}$ Perryman, M. A. C. and van Leeuwen, F., editors, The Gaia Concept, Vol. 379 of ESA Special Publication, 1995.

${ }^{18}$ Jordan, S. and Jäger, K., "Gaia - Teamwork for a Billion Stars," https://www . youtube.com/watch?v=E9GYLcesPp0, Apr 2016, [Online; accessed 19-May-2016].

${ }^{19}$ Lefferts, E. J., Markley, F. L., and Shuster, M. D., "Kalman filtering for spacecraft attitude estimation," Journal of Guidance, Control, and Dynamics, Vol. 5, No. 5, 1982, pp. 417-429.
} 
${ }^{20}$ Markley, F. L., "Attitude error representations for Kalman filtering," Journal of guidance, control, and dynamics, Vol. 26, No. 2, 2003, pp. 311-317.

${ }^{21}$ Crassidis, J. L. and Junkins, J. L., Optimal estimation of dynamic systems, CRC press, 2011.

${ }^{22}$ Titterton, D. and Weston, J. L., Strapdown inertial navigation technology, Vol. 17, IET, 2004.

${ }^{23}$ Trawny, N. and Roumeliotis, S. I., "Indirect Kalman filter for 3D attitude estimation," University of Minnesota, Dept. of Comp. Sci. \& Eng., Tech. Rep, Vol. 2, Mar 2005.

${ }^{24}$ Burnside, J. W., Murphy, D. V., Knight, F. K., and Khatri, F. I., "A Hybrid Stabilization Approach for Deep-Space Optical Communications Terminals," Proceedings of the IEEE, Vol. 95, No. 10, Oct 2007.

${ }^{25}$ Biswas, A., Hemmati, H., Piazzolla, S., Moision, B., Birnbaum, K., and Quirk, K., "Deep-space optical terminals (DOT) systems engineering," Interplanetary Network Progress Report, Vol. 42, 2010.

${ }^{26}$ Chen, C.-C., Hemmati, H., Biswas, A., Ortiz, G., Farr, W., and Pedreiro, N., "Simplified lasercom system architecture using a disturbance-free platform," 2006.

${ }^{27}$ University of Wisconsin-Madison Space Astronomy Laboratory, ST5000, 2007, [Online; accessed 10-Aug-2016].

${ }^{28}$ Gyro and Accelerometer Panel of the IEEE Aerospace and Electornic Systems Society, "IEEE Standard Specification Format Guide and Test Procedure for Single-Axis Interferometric Fiber Optic Gyros," Tech. Rep. Std 952-1997 (R2008), IEEE, 2008.

${ }^{29}$ NASA, "Wallops Arc Second Pointer (WASP)," NP-2013-10-069-GSFC, 2013.

${ }^{30}$ Northrop Grumman Systems Corporation, Woodland Hills, CA, Scalable SIRU, 2013, [Online; accessed 20-Nov-2014].

${ }^{31}$ Meyer, D. and Rozelle, D., "Milli-HRG inertial navigation system," Gyroscopy and Navigation, Vol. 3, No. 4, 2012. 